\section{RICE BREEDING}

\section{Signature analysis}

Proc. Natl Acad. Sci. USA http://doi.org/7st (2015)

Recently large-scale population genomic analyses have been effectively applied in the study of rice domestication. However, this powerful tool has yet to be sufficiently employed in the study of modern rice breeding. Weibo Xie and colleagues from the Huazhong Agricultural University have now unravelled the breeding signatures of indica rice by analysing the genomes of 1,479 rice accessions.

Using the genomic data from two previous studies, the researchers obtained over six million single nucleotide polymorphisms (SNPs) classifying indica rice into two groups - IndI and IndII. About $37 \%$ of the IndI accessions and 95\% of the IndII accessions are semi-dwarf improved cultivars, corresponding to two heterotic groups. The founder lines of the two heterotic groups were inferred to be Aijiaonante and IR8, respectively.

Selective sweep analysis showed that 200 regions, covering $7.8 \%$ of the rice genome, have been differentially selected in the two groups, some of which overlap with domestication-related regions.

Genes related to important agronomic traits - such as plant architecture, disease resistance and grain yield - are found in these regions.

A significant correlation was observed between yield and the number of selected SNP alleles or haplotypes in an accession in both the indica population and another two artificial segregating populations. These selected alleles or haplotypes are breeding signatures that can be used to predict agronomic potential and facilitate rice improvement.

\section{FOREST ECOLOGY}

Three trillion trees

Nature 525, 201-205 (2015)

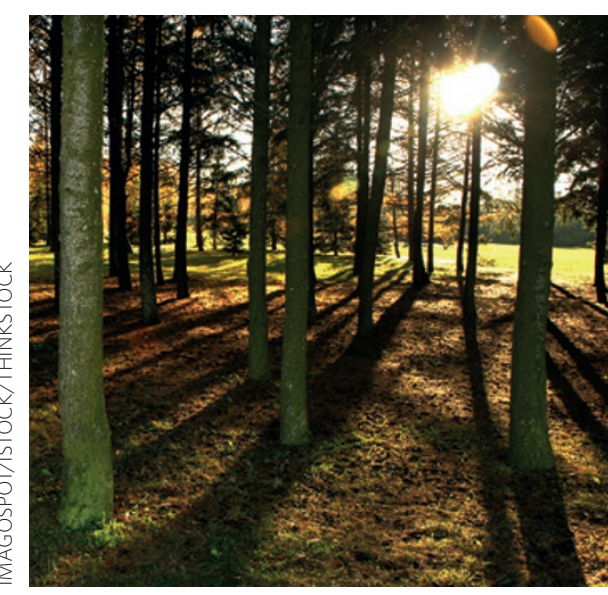

The planet is home to an order of magnitude more trees than previously thought, reveals a global study of tree density.

Thomas Crowther, of Yale University, and colleagues mapped forest tree density at a global scale, using tree count data from every continent on Earth barring Antarctica, satellite imagery and biomespecific regression models. The total number of trees on the planet amounts to around 3.04 trillion according to their
FLOWER COLOUR

\section{Peach cocktail}

J. Exp. Bot. http://doi.org/7sn (2015)

Variegation - the presence of different coloured sectors within a single flower or leaf - is a highly valued horticultural trait. Transposable elements are most commonly responsible; unstably disrupting genes involved in pigment production to generate variegated varieties of flowers such as petunia, antirrhinum, carnations, roses and morning glories. However, transposable elements are not the only route to variegation as Yuepeng Han and colleagues at Wuhan Botanical Garden amply demonstrate.

The researchers investigated the ornamental peach variety Hongbaihuatao. Flowers on a single tree can be red, pink, white or variegated combinations of all three. HPLC analysis of petals showed that the variation in colour was due to differing levels of the anthocyanin cyanadin 3-glucoside, which is abundant in red petals but absent in white. The intermediate pink regions arose when the anthocyanin was confined to the upper and lower epidermal cell layers only.

Well over fifty genes were differentially expressed between red and white regions however only two encoded proteins involved in anthocyanin production and only one, a glutathione $S$-transferase called Raist, showed sequence differences corresponding to different flower colours. In white petals a two-base-pair insertion created a premature STOP codon. Although no transposable elements play a part in this alteration of Raist, the mechanism by which these spontaneous insertions occur remains to be determined. calculations, equivalent to 422 trees per person. The greatest densities are found in the boreal forests of North America, Russia and Scandinavia. But the greatest numbers are found in tropical and subtropical regions, which together harbour the highest proportion of the world's forested land, and over $40 \%$ of the world's trees.

Combining their density data with a spatially explicit map of forest cover loss, the researchers estimate that deforestation, forest management, disturbance and land use change are collectively leading to the loss of over 15 billion trees each year. A comparison of their data with estimates of ancient forest cover suggests that since the onset of agriculture the global number of trees has fallen by over $45 \%$.

\section{IMMUNITY SIGNALLING}

\section{The WRKY connection}

\section{Plant Cell http://doi.org/7sp (2015)}

Fast activation of a central MAP kinase cascade and the production of reactive oxygen species (ROS) by membrane NADPH oxidases (known as RBOHs) are two common responses to multiple biotic and abiotic danger signals. Adachi et al. now show that both are connected in immune responses through so-called 'WRKY' transcription factors.

The ROS response to pathogens is biphasic. The fast and transient first stage is specific to pattern-induced immunity, posttranslationally regulated by calcium and the kinase BIK1, which directly and functionally connects immune receptors with Arabidopsis RBOHD. The slow and sustained second stage is activated transcriptionally, specific to effector-induced immunity, and important for the hypersensitive response that limits pathogen spread.

The authors show that four WRKYs are substrates of the activated MAPKs in tobacco. Once phosphorylated, these transcription factors will directly bind and activate the promoter of tobacco $\mathrm{RBOHB}$ and produce the secondary ROS burst needed for the hypersensitive response. The increased susceptibility to oomycete Phytophthora infestans of a plant with silenced WRKYs confirms the biological significance of this mechanism. These results are a significant step toward unravelling the role of pathways downstream of MAPK cascades in defence, and maybe extending this understanding to further danger signals.

Written by Anna Armstrong, Jun Lyu, Chris Surridge and Guillaume Tena. 